\title{
Conference Scene
}

UK Pharmacogenetics and Stratified Medicine Network

\section{UK Pharmacogenetics and Stratified Medicine Network Conference 2011 Wellcome Trust Genome Campus, Hinxton, Cambridge, UK, 28 September 2011}

The second Annual Meeting of the UK Pharmacogenetics and Stratified Medicine Network was held on 28 September 2011 at The Wellcome Trust Conference Centre on the Wellcome Trust Genome Campus at Hinxton, Cambridge, UK. The meeting preceded the annual Pharmacogenomics and Personalized Medicine conference, which was held over the subsequent two days. Around 95 individuals from a wide range of backgrounds including research councils, academia and industry attended the meeting.

Spoken presentations in the afternoon concentrated on potential funding mechanisms within the UK for stratified medicine research, including information on dedicated income streams from National Institute for Health Research (NIHR) and the Technology Strategy Board. The potential for stratified medicines to form a focus for small/medium enterprise activity was highlighted by Alun McCarthy of PGXIS (UK) who highlighted that $15 \%$ of EU Framework 7 programme (FP7) funding is ring fenced for small/medium enterprises.

John Whittaker (GlaxoSmithKline, UK) gave a presentation on how stratified medicine approaches may assist in drug development from the perspective of a major pharmaceutical company. He highlighted the potential value of these approaches both for efficacy and adverse drug event identification, and also the potential for defining high-risk populations in clinical studies which might improve efficiency for clinical trial design. Examples he described included lapatinib, a HER2/EGFR tyrosine kinase inhibitor used in HER2-positive breast cancer. A small number of individuals developed severe liver injury with this agent and there is a strong association with $H L A D Q A 1^{*} 0201$ haplotype. However, because this haplotype is relatively common in the general population, excluding all individuals from treatment would result in many individuals who would potentially benefit failing to receive treatment. The current approach is to try and refine the signal using next-generation sequencing approaches with the aim of identifying individuals who might require enhanced liver-function test monitoring. He also described work on pazopanib, a drug in development for the treatment of age-related macular degeneration. This is a condition in which a relatively large number of genetic risk variants have been identified and hence there is potential to stratify populations to include those most at risk based on genetic profiles and to improve the efficiency of clinical trials. IL8 SNPs have been associated with efficacy and variation in a number of other genes including: VEGF, CFH and ARMS2, as well as genes in the complement cascade which can potentially be used to optimize trial design. However, experience to date suggests that genotyping adds relatively little to simple clinical scoring systems and hence, any reduction in trial size from genetic analyses is likely to be, at best, small.

The theme of clinical trial design was also explored by Mahesh Parmar from the MRC's Clinical Trial Unit. Mahesh emphasized how clinical trial design needs to be 'reactive' to new approaches particularly where studies are likely to be long term because of the need to be able to adapt to new discoveries. He discussed designs to deal with multiple study arms in a large trial in metastatic colorectal cancer (FOCUS 4) using biomarkers for treatment stratification.

In addition to formal presentations a number of poster presentations were given including an update of Swedegene by Mia Wadelius from Uppsala. This interesting project aims to collect a Biobank from individuals suffering adverse drug reactions to facilitate genetic or other biomarker research. The target group of adverse drug reactions include drug-induced liver reactions, severe skin reactions, agranulocytosis, long-QT syndrome, CNS toxicity,

\section{Ian Hall}

University of Nottingham Medical School, Nottingham, NG7 2UH, UK

ian.hall@nottingham.ac.uk 
statin myopathy, fluoroquinolone-induced tendon rupture, angioedema and cough related to angiotensin-converting enzyme inhibitors, osteonecrosis due to bisphosphonates, weight gain due to neuroleptics and hyponatremia due to antidepressants and antiepileptic drugs. To date, over 400 cases have been collected, although the number of individuals with any specific phenotype remains small at present and the group is interested in establishing new collaborations in addition to those already in place within the Eudragene network.

The UK Pharmacogenetics and Stratified Medicine Network is a young organization; however, it has the potential to provide an extremely valuable support network within the UK and internationally. With this in mind, it was particularly useful to hear the perspective from Teri Klein (University of Stanford, CA, USA) whose experience over the last 12 years with the Pharmacogenomics Knowledge Base [101] was invaluable. This started out as the NIH Pharmacogenetics Research Network (PGRN) with a model including network-wide projects in 14 research groups and seven network research centers with expertise such as exome sequencing. The budget for this was approximately US\$33 million per year and Teri's view was that without this level of infrastructure support, the impressive outputs of PharmGKB and PGRN would not have occurred. To date, the two organizations have contributed to approximately 1000 academic publications, of which almost 25 were authored specifically by a network. In addition to the academic output and the resources provided by the network, there was a move towards provision of clinical information through the CPIC consortium, which has helped provide guidance on the use of stratified approaches in the prescribing of drugs including warfarin, tamoxifen, clopidogrel and selective serotonin reuptake inhibitors.

In summary, this was a useful meeting for all those interested in stratified medicine approaches, both from a research point of view and for those interested in service delivery. The key challenge for the UK now will be to take forward this agenda, which will require adequate resourcing, in order to improve clinical care, patient safety and treatment outcomes.

\section{Financial \& competing interests disclosure}

The author has no relevant affiliations or financial involvement with any organization or entity with a financial interest in or financial conflict with the subject matter or materials discussed in the manuscript. This includes employment, consultancies, honoraria, stock ownership or options, expert testimony, grants or patents received or pending, or royalties.

No writing assistance was utilized in the production of this manuscript.

\section{Website}

101 Pharmacogenomics Knowledge Base. www.pharmgkb.org 